\title{
LA INCUESTIONABILIDAD DEL RIESGO: VULNERABILIDAD SOCIAL Y RIESGO SÍSMICO EN MUNICIPIOS TURÍSTICOS
}

\author{
Antonio Aledo \\ Universidad de Alicante \\ Samia Sulaiman \\ Universidad de Alicante y Universidad de São Paulo
}

\section{RESUMEN}

Los estudios sobre la vulnerabilidad social han ampliado la gestión de los riesgos naturales tradicionalmente direccionada a la amenaza natural y soluciones tecnológicas. Sin embargo la visión dominante se mantiene hegemónica sin un análisis adecuado de las causas que originan vulnerabilidad social.Este artículo propone un nuevo argumento, organizado en torno al concepto de la incuestionabilidad del riesgo, entendido como la incapacidad cultural y política de objetar el modelo socioeconómico imperante en donde residen las causas de fondo productoras de riesgo. Para concretar el concepto, a modo de ejemplo, se analizan el Plan de Actuación Municipal frente al Riesgo Sísmico de la ciudad de Torrevieja.

Palabras clave: Gestión de riesgos socio-naturales; visión dominante; turismo residencial; España; terremotos.

The unquestionability of risk: social vulnerability and earthquake risk in tourist destinations

\section{ABSTRACT}

The studies on environmental risk have been traditionally focused on natural hazards and technological solutions, but they have recently broadened their scope with the introduction of social vulnerability into the research agenda. However, the dominant view

Recibido: 17 de abril de 2014

Devuelto para su revisión: 10 de julio de 2014

Aceptado: 15 de septiembre de 2014

Departamento de Sociología I. Universidad de Alicante. Apartado 99. 03080 ALICANTE (España). E-mail: antonio.aledo@ua.es, samia.sulaiman@gmail.com 
is still hegemonic. This article proposes a new explanatory element,built around the concept of unquestionability of risk. It refers to the cultural and political inability to challenge the prevailing socio-economic model that is the origin of the causes of risk production. In order to illustrate this idea, the Local Plan against Seismic Risk of the municipality of Torrevieja (Southeastern Spain) is analyzed.

Key words: Socio-environmental risk management; dominant view; residential tourism; Spain; earthquakes.

\section{PRESENTACIÓN}

Desde la década de los 80, la literatura especializada en el análisis y gestión de riesgos naturales muestra un desplazamiento hacia el enfoque de la vulnerabilidad (Blanchard, 2000). Previamente a este giro, la visión dominante o tecnocrática (Hewitt, 1983; Phillips y Fordham, 2010: 6-12) establecía que los desastres naturales eran sucesos físicos extremos, producidos por una naturaleza caprichosa, externos a lo social y que requerían soluciones tecnológicas y de gestión de expertos (Bankoff, Frerks y Hilhorst, 2004; Cannon, 1994; Quarantelli, 1998; Tobin y Montz, 1997). Esta visión dominante comenzó cuestionarse al reconocer, por un lado, el papel que juega la vulnerabilidad social $^{1}$ en la producción del riesgo (Hewitt, 1983; Wisner et al. 2004) y, por otro, que las soluciones exclusivamente tecnológicas eran insuficientes, ineficaces y, muchas veces, contraproducentes (Beck, 1992).

La visión de la vulnerabilidad ante los riesgos ambientales entiende que a) los desastres son fenómenos socio-naturales (unnatural disasters) (Abramovitz, 2001); b) por lo tanto, en su producción también actúan factores socio-históricos (Pelling, 2003; Steinberg, 2000; Tobin and Montz, 1997); y c) que sus efectos se distribuyen de forma desigual entre la población (Bara, 2010; Klinenberg, 2002). Siguiendo el trabajo seminal de Wisner et al (2004: 9), se entiende por vulnerabilidad las características de una persona o grupo desde el punto de vista de su capacidad para anticipar, sobrevivir, resistir y recuperarse del impacto de una amenaza natural. Por tanto el riesgo es producto no solo de una amenaza sino de las condiciones de vulnerabilidad de la población y del territorio (Wisner, 2000).

No obstante, y aquí reside el punto de partida de este artículo, una parte importante de esta misma literatura reconoce que, en la práctica de la planificación y gestión de los riesgos naturales, la visión dominante sigue ocupando una posición hegemónica. Juneja habla de un conceptual lag (2009: 2) aludiendo, por un lado, a la aceptación entre los claim-makers $^{2}$ del riesgo ${ }^{3}$ del discurso que alaba las ventajas de un enfoque integrado que

1 En este artículo cuando se hable de vulnerabilidad se entenderá siempre como vulnerabilidad social. Si se hace referencia a otro tipo de vulnerabilidades - económica, constructiva, etc.- irá acompañada del adjetivo correspondiente.

2 Los claim-makers (Stallings, 1995) son técnicos, expertos, científicos o funcionarios que se sitúan en la gestión del riesgo a escala meso y/o local.

3 La literatura especializada aporta una amplia variedad de definiciones de vulnerabilidad. Los diferentes enfoques teóricos, metodológicos, prácticos e incluso ideológicos desde los que los expertos se aproximan a su 
resalte la vulnerabilidad y, por otro, la persistencia de la visión dominante en la práctica de la gestión del riesgo que privilegia soluciones tecnológicas, top-down y que minimiza el análisis y tratamiento de las causas que originan vulnerabilidad social. Es importante entonces analizar los factores que intervienen en la reproducción de esta situación que se podría calificar casi como esquizofrénica.

El objetivo de este artículo se orienta a proponer un nuevo componente explicativo a la persistencia hegemónica de la visión tecnocrática. Este argumento se ha organizado en torno al concepto de la incuestionabilidad del riesgo. Sus fuentes teóricas se encuentran en las perspectivas sociológicas sobre análisis del riesgo (ver Renn, 2008) y en las teorías del post-ecologismo (Blühdorn, 2000; Blühdorn y Welsh, 2007). De forma breve, la incuestionabilidad del riesgo se refiere a la incapacidad cultural y política de objetar el modelo socioeconómico imperante en donde residen las causas de fondo productoras de riesgo.

A fin de mostrar el papel que juega la incuestionabilidad del riesgo en la orientación de los planes y prácticas de gestión del riesgo ambiental y las consecuencias que conlleva, se toma como caso de estudio la ciudad de Torrevieja, España, y las directrices que orientan la construcción de su Plan de Actuación Municipal frente al Riesgo Sísmico (PAM). El riesgo sísmico en el municipio de Torrevieja ejemplifica perfectamente la ecuación de Wisner et al. (2004) de que el riesgo es producto de la conjunción de la amenaza sobre una población en situación de vulnerabilidad. Esta localidad se encuentra en una zona de alta peligrosidad sísmica y ha experimentado un rápido y desordenado crecimiento urbanístico ligado al fenómeno del turismo residencial que ha propiciado la aparición de condiciones de vulnerabilidad entre la población. Además, a partir de 2012, el Consistorio ha puesto en marcha la planificación y gestión ante el riesgo sísmico, siguiendo la visión dominante que, según la argumentación que se desarrolla en este artículo, no pone en cuestión el modelo socioeconómico productor de riesgo.

El orden argumental que seguirá este artículo es el siguiente. Primero, se llevará cabo una caracterización comparativa entre la visión dominante y la perspectiva de la vulnerabilidad en la gestión del riesgo. Segundo, se repasarán las causas que la literatura especializada ha señalado como principales factores explicativos de la persistencia de la visión dominante. Tercero, se adentrará en el concepto de la incuestionabilidad del riesgo, su justificación axiológica y sus fuentes teóricas. Cuarto, se describirá el caso de estudio presentando, por un lado, el modelo de desarrollo socio-urbanístico de Torrevieja y, por otro, el PAM frente al Riesgo Sísmico. Quinto, se discutirán los datos presentados en el punto anterior mostrando la pertenencia del PAM a la visión dominante al centrarse en

análisis ocasionan esta dispersión terminológica. En aras de una mayor precisión, algunos autores han establecido una diferencia entre exposición y vulnerabilidad. Según la Estrategia para la Reducción de Desastres de Naciones Unidas (UNISDR, 2009: 17), el grado de exposición significa: «La población, las propiedades, los sistemas u otros elementos presentes en las zonas donde existen amenazas y, por consiguiente, están expuestos a experimentar pérdidas potenciales», mientras que vulnerabilidad involucra «las características y las circunstancias de una comunidad, sistema o bien que los hacen susceptibles a los efectos dañinos de una amenaza». De manera analítica, Cutter et al (2003) apunta que tradicionalmente la vulnerabilidad ha sido considerada una condición preexistente o exposición potencial al riesgo (vulnerabilidad biofísica) o una condición social que indica una predisposición de respuesta a una amenaza natural (vulnerabilidad social y propone una «tercera via» la vulnerabilidad como «amenaza del lugar» que relaciona tanto la vulnerabilidad biofísica como la social, sometidas al análisis del territorio. 
soluciones tecnológicas y en las expresiones más superficiales de la vulnerabilidad, esquivando el análisis y cuestionamiento de las causas de fondo que originan la vulnerabilidad en este municipio. Por último, se concluirá con una reflexión sobre qué aportaciones puede hacer a la gestión del riesgo el enfoque seguido en este artículo en la búsqueda de un equilibrio integrador y propositivo al situarse entre las investigaciones generadoras de conocimiento crítico que se mueve a niveles macro y meso y las necesidades de propuestas prácticas susceptibles de ser implementadas en los planes de gestión del riesgo, el enfoque que se ha denominado «Compensatory Disaster Risk Management» (UNDP, 2004; citado en Bara, 2010: 17).

\section{LA VISIÓN DOMINANTE FRENTE A LA PERSPECTIVA DE LA VULNERA- BILIDAD}

Para explicar la persistencia hegemónica de la visión dominante frente a la sumisión de la perspectiva de la vulnerabilidad se precisa previamente comparar ambas visiones de los desastres naturales. Desde la visión dominante, la naturaleza es entendida como una fuerza externa causante de los desastres ambientales (Hewitt, 1983; Tobin y Montz, 1997). El ser humano puede imponer su dominio sobre esa naturaleza caótica y amenazante mediante la ciencia, la tecnología, las ingenierías y las técnicas de gestión (Phillips y Fordham, 2010). El manejo de estos instrumentos se realiza de forma jerarquizada, por parte de las autoridades y expertos, con un enfoque top-down (Blanchard, 2000). El objetivo final es doble: 1) reducir los impactos a través de acciones de detección preventiva, construcción de obras de infraestructura, mitigación, adaptación y rehabilitación y 2) recuperar el orden social dislocado por el efecto dañino del evento ambiental (Quarantelli, 1998).

Esta visión se ha visto contestada por el surgimiento de la perspectiva de la vulnerabilidad que enfatiza los entornos sociales, políticos y económicos que intervienen en el proceso causativo de los desastres (Wisner et al., 2004: 4). La perspectiva de la vulnerabilidad insiste en diferenciar la amenaza (hazard) del desastre (Quarantelli, 1985). Se enfatiza las influencias socioeconómicas y políticas asociadas a los desastres (Wisner et al., 2004, Ayala-Carcedo y Olcina, 2002). Propone una gestión descentralizada y basada en soluciones locales generadas en procesos participativos con un enfoque bottom-up (Blanchard, 2000; Enarson et al., 2003). De esta manera reconoce la relevancia del conocimiento local para mejorar la gestión de los desastres. Apela a valores de igualdad social y de cooperación con la naturaleza. Prefiere hablar de sistemas socio-naturales y los entiende como abiertos y complejos. Introduce el manejo de la incertidumbre (Gilbert, 1995) como una variable y, por tanto, cuestiona las certezas propuestas por el modo de conocer de la ciencia occidental neopositivista.

\section{LA HEGEMONÍA DE LA VISIÓN DOMINANTE}

Numerosos autores señalan que a pesar de la insistencia discursiva en las virtudes del enfoque de la vulnerabilidad, la práctica de la gestión del riesgo sigue estando dominada por la visión hegemónica (Burton, Kates y White, 1993; McEntire, 2003; Mileti, 1999; Tierney, 2007). Las razones que pueden ayudar a entender esta preponderancia se han 
agrupado en explicaciones culturales y estructurales. La visión dominante participa del paradigma occidental antropocéntrico (Sheldrake, 1994) que sitúa al ser humano como una especie distinta y superior al resto, con derecho a dominar la naturaleza, y con una confianza ilimitada en la ciencia y la tecnología para cumplir este objetivo de control (Dryzec, 2005). Los desastres serían entonces carencias o errores del proyecto modernista de controlar la naturaleza. La respuesta ante los desastres solo puede ser, bajo este paradigma, mejorar y ampliar los procesos científico-tecnológicos de producción de conocimiento, de control, predicción, de gestión y respuesta ante los efectos externos -no sociales- del medio ambiente. Por otro lado, las explicaciones de carácter estructural (Scandlyn et al., 2010: 43-44) hacen referencia a los beneficios económicos y de otro tipo que obtienen determinados grupos o lobbies -ligados a la construcción de grandes obras públicas o a la producción de tecnología predictiva- de mantener la aplicación de la visión dominante en la gestión de los desastres (Alexander, 2000: 25). También una parte importante de las ciencias apoya esta visión (Phillips y Fordham, 2010: 8), ya sea por la inercia intelectual del neopositivismo (Blanchard, 2000) y/o por las ventajas académicas y económicas que obtienen de la investigación en monitoreo geofísico, predicción, diseño de obras de ingeniería u ordenación del territorio (Hewitt, 1983: 5).

\section{LA INCUESTIONABILIDAD DEL RIESGO}

Además de estas razones, este artículo presenta un tercer argumento que se ha denominado la incuestionabilidad del riesgo. En los siguientes párrafos se argumenta esta línea al plantearse la incuestionabilidad del riesgo como la incapacidad y desidia de los expertos, científicos y tomadores de decisiones en general (claim-makers) de identificar y actuar sobre las causas profundas de la producción del riesgo ya que ello conllevaría a cuestionar los imperativos normativos, las necesidades de las elites y los estilos de vida del actual sistema socioeconómico globalizado.

Antes habría que hacer una referencia al concepto de causas profundas del riesgo que se ha obtenido del trabajo de Wisner et al. (2004: 27-28) sobre el Modelo de Presión y Liberación. Este modelo propone una perspectiva de progresión de la vulnerabilidad en tres niveles de identificación: 1) las «causas de fondo» que resultan en 2) «presiones dinámicas» y se materializan en los entornos locales por medio de 3) «condiciones inseguras». Las causas de fondo están ligadas a la morfología de las estructuras ideológicas, económicas, sociales y legales, y los tipos de relaciones de género, entre clases o etnias. Las causas de fondo reflejan la desigual distribución del poder y son reproducidas a través de discursos hegemónicos que legitiman y normalizan estas estructuras desiguales.

El concepto de incuestionabilidad del riesgo puede entenderse mejor a partir de las perspectivas sociológicas sobre análisis del riesgo (ver Renn, 2008) y en las teorías del postecologismo (Blühdorn, 2000; Blühdorn y Welsh, 2007; Blühdorn, 2009). El actual sistema socioeconómico se sostiene sobre tres imperativos: 1) el crecimiento continuo erigido sobre el control de la naturaleza; 2) la ciencia y tecnología que faculta a los expertos el control de sistemas cada vez más complejos; y 3) el mercado como el agente capaz de gestionar de forma sostenible las relaciones entre sociedad, economía y medio ambiente. Siguiendo a Blühdorn (2009) estos tres imperativos se han convertido en universales e incuestionables. 
Sin embargo, y esta es la contradicción central en la que ha caído el mito del progreso moderno, la triada crecimiento continuo, ciencia/tecnología y mercado son productores de insostenibilidad y riesgo y, por tanto, terminan por socavar el propio sistema.

Si Schnaiberg, en su conocida obra Environment: from surplus to scarcity (1980), reveló que la lógica inherente del capitalismo requiere el crecimiento constante, la obra seminal de Georgescu-Roegen The Entropy Law and The Economic Process (1971) demostró la imposibilidad del crecimiento continuo en un mundo antrópico. La consecuencia directa de esta contradicción es la degradación del ecosistema, el agotamiento de los recursos y el aumento de la contaminación en sus diversas formas (Millennium Ecosystem Assessment, 2005). Por su parte, los estudios de Ecología Política (Bryant y Bailey, 1997; Robbins, 2004) subrayan cómo el mercado provoca que dicha degradación se distribuya desigualmente por la geografía espacial y social mundial. Por último, las teorías sociológicas del riesgo (Bauman, 1996; Beck, 1992) muestran cómo este es producto del propio desarrollo tecnológico (Laraña, 1999) una vez que ha escapado al control social y se deja guiar exclusivamente por los dictados del mercado. En definitiva, el riesgo no es un error ni siquiera una inevitable consecuencia del progreso. El riesgo es consustancial a la alta modernidad, su otra cara (Giddens, 1990).

Así, la sociedad se enfrenta, por un lado, al riesgo de una crisis ambiental global y, por otro, a la imposibilidad de cuestionar las bases, el modo de producción, que genera ese riesgo global. Para Blühdorn (2000) esta contradicción nos sitúa en la era del postecologismo. Los imperativos sistémicos y los estilos de vida adquieren un estatus no negociable. Todo aquello que cuestione el crecimiento continuo, la capacidad de control de la ciencia y tecnología y el mercado será calificado como utópico en su crítica light o de anti-sistema en su definición de la realidad más coercitiva. El giro postecologista significa no solo la victoria de las políticas neoliberales o un paso más en el proceso de alienación de los ciudadanos para transformarlos en masas de consumidores, sino también el dominio de la aproximación tecnológica y de gestión, lo que implica definiciones de los problemas ambientales estrechas y cortoplacistas, vaciadas de contenidos políticos y sin visiones ecosistémicas alternativas al modelo socioeconómico dominante.

Las decisiones que se acuerdan en la gestión del riesgo, se dirigen, en el mejor de los casos, a actuar sobre el control o manejo tecno-científico de la amenaza y a intentar contraer o minimizar las condiciones más superficiales y locales de la vulnerabilidad (condiciones inseguras). Los gestores, los expertos y científicos, instalados en la visión dominante, proponen la construcción de obras de infraestructuras o diseñan tecnologías altamente complejas para la prevención, mitigación y monitoreo de la amenaza. Y las acciones de educación se destinan a la preparación de la respuesta de la población ante el desastre. En el peor de los casos, aparece un discurso que desestima y desacredita los escenarios de riesgo ambiental escudándose en la necesidad de superar una crisis económica que paradójicamente ha impulsado el propio modelo. Así los factores de producción de vulnerabilidad y riesgo relacionados con estructuras sociales desiguales, con fuertes concentraciones de poder o con modelos de desarrollo insostenibles son sutilmente soslayados para continuar con un modelo socioeconómico que se ha diseñado para concentrar los beneficios en cada vez menos manos y, por el contrario, para socializar las externalidades ambientales y sociales que provoca. 
Dibujado el concepto, en las siguientes páginas se ejemplificará la incuestionabilidad del riesgo analizando el plan municipal ante el riesgo sísmico en el municipio de Torrevieja, España.

\section{EL CASO DE LA GESTIÓN DEL RIESGO SÍSMICO EN TORREVIEJA}

Esta localidad de la Costa Blanca (Alicante-España) representa un caso especialmente adecuado para analizar el proceso de incuestionabilidad del riesgo debido a: 1) su localización en una zona de alto riesgo sísmico; 2) un modelo socio-urbanístico centrado en el sector turístico residencial caracterizado por su rápido crecimiento que ha intensificado la producción de vulnerabilidad; y 3) el inicio, por parte de los técnicos municipales, de un plan ante el riesgo sísmico. A partir de su análisis bajo el concepto de incuestionabilidad del riesgo, se plantea que el Plan de Actuación Municipal (PAM) frente al Riesgo Sísmico de Torrevieja es funcional en el marco de los presupuestos ideológicos del postecologismo, en tanto en cuanto: 1) está diseñado para reforzar el papel de los técnicos locales; 2) apacigua la alarma social producida por el cercano terremoto de Lorca de 2011, 3) responde al nuevo marco jurídico para la gestión de riesgos naturales, y 4) the last but not the least, no cuestiona el modelo de desarrollo socio-urbanístico donde, en última instancia, reside la producción de la vulnerabilidad.

En cuanto a la metodología, este trabajo ha seguido un diseño de investigación emergente, de base exploratoria y explicativa, para dar cuenta de una relación causa-efecto entre la realidad socio-histórica y la producción de vulnerabilidad. Se entiende que un estudio de caso no proporciona elementos de extrapolaridad ni pretende ser representativo de la praxis social de la visión dominante ante los desastres. Un estudio de caso se selecciona por su capacidad de ilustrar un proceso y ayudar a entender una teoría. En este caso, se emplea para describir las causas y consecuencias del predominio de la visión dominante en la gestión de los riesgos ambientales y ejemplificar el concepto de incuestionabilidad del riesgo.

Se ha realizado una revisión bibliográfica sobre la amenaza sísmica en el área de estudio, la ocupación humana y los usos del suelo de Torrevieja, y el marco jurídico español sobre ordenación del territorio, urbanismo y protección civil. Además, se ha realizado una recolección de información por medio de entrevistas semi-estructuradas con informantes clave procedentes de tres ámbitos: a) ámbito académico-investigativo; b) cuadros técnico-administrativo relacionados con los planes de actuación locales y regionales frente a riesgos naturales y c) miembros de partidos políticos y ONGs encargados de la gestión o seguimiento del PAM. Para la triangulación de las informaciones y datos obtenidos, se ha contado con datos secundarios estadísticos de los Censos de Población y Vivienda y del Padrón Municipal de Torrevieja.

\subsection{La amenaza sísmica}

Torrevieja está ubicada en el Sistema Tectónico Bético-Rifeño-Mar de Alborán, dentro de un área sísmica denominada Íbero-Magrebí, y asociada al límite entre las placas Africana y Euroasiática, que convergen en la dirección noroeste-sureste con una velocidad 
de aproximadamente 5mm/año (PELSISCAV, 2010). A nivel regional destaca la Falla del Bajo Segura, una de las fallas más activas de la Península Ibérica durante los últimos 500 años, sobresaliendo la serie sísmica de 1829, conocida como el Terremoto de Torrevieja, que alcanzó intensidad IX-X, relativo a un terremoto destructor en la Escala Macrosísmica Europea (EMS-98) (Fig. 1). En esta región está localizado el núcleo sísmico de Torrevieja, al que las estimaciones de intensidad sísmica máxima esperada llegan a 9,5 (EMS-98), que representa un terremoto de efecto destructor, según el Estudio de Peligrosidad Sísmica de la Comunitat Valenciana (PELSISCAV, 2010).

\section{Figura 1}

\section{MAPA DE INTENSIDADES PROMEDIO ESPERADAS \\ EN LA COMUNIDAD VALENCIANA}

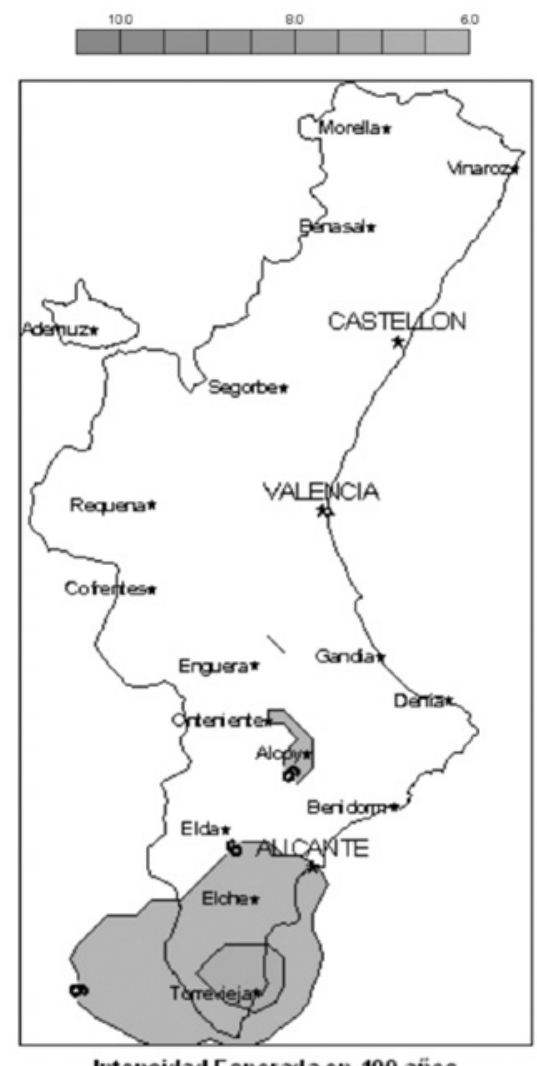

Intensidad Esperada en 100 años

Fuente: Giner Caturla (1996).

Situada en la costa, con una franja litoral de $13,25 \mathrm{~km}$ y presentando $55 \%$ del territorio ocupado por extensiones lagunares, Torrevieja posee características litológicas que pueden modificar la respuesta del suelo ante un terremoto. Los limos de las lagunas, por ejemplo, 
han sido evaluados como materiales de peor respuesta frente a una sacudida sísmica, por comportarse de forma semejante a un fluido, resultado característico de la licuefacción (Giner, Mateo y Estévez 1992). Según el PELSISCAV, si se considera el efecto suelo, para periodos de retorno de 500 y 1000 años, se aumenta de 0.5 a 1.0 el grado de las intensidades sísmicas máximas esperadas, pasando la probabilidad de un terremoto dañino (intensidad VII) a uno muy destructor (intensidad X).

El mencionado terremoto de 21 de mayo de1829 fue el más devastador del sureste de la Península Ibérica en los últimos 500 años (Lázaro, 1829). Después de ese evento catastrófico, el ingeniero de caminos José Larramendi propuso una serie de medidas urbanoarquitectónicas para la reconstrucción de la ciudad (Larramendi, 1829 a, b ). El nuevo núcleo se diseñó sobre plano hipodámico y con un modelo arquitectónico de viviendas de planta baja, con patios o corrales interiores con función de refugio, edificaciones más resistentes, con fuerte trabado entre los principales elementos estructurales, ausencia de adornos y sin cornisas (Calvo García-Tornel y Canales Martínez; 2009).

A partir de la década de los sesenta del siglo XX, Torrevieja experimentó una profunda transformación de su base económica. Esta mudanza ha conllevado un incremento del área urbanizada y de la densidad edificatoria en un corto periodo de tiempo, y el olvido del modelo urbano-arquitectónico, adaptado a la amenaza sísmica de la región (Canales Martínez, 1999). A partir de los años setenta la economía local, que se basaba en la actividad salinera y la pesca, dejó paso al monopolio de la función turístico-residencial, convirtiéndose en uno de los principales destinos turísticos del Mediterráneo (Casado-Diaz, 1999).

\subsection{El nuevo modelo socio-urbanístico}

El modelo turístico residencial implementado en Torrevieja se caracteriza por su altísima producción inmobiliaria, por su elevada estacionalidad, por la falta de planeamiento urbanístico, por la degradación ambiental, por promover un cambio demográfico debido a altas tasas de inmigración y envejecimiento y por ir asociado a una gestión del urbanismo municipal opaca y ligada a varios y llamativos casos de corrupción. Su producto entremezcla la oferta de sol y playa junto con decenas de miles de viviendas turístico-residenciales construidas en torno a urbanizaciones orientadas a los mercados de jubilados extranjeros y nacionales y familias de veraneantes (Aledo, Mazón y Mantecón, 2007).

El sector turístico residencial se basa en la continua construcción y venta de viviendas. Esta característica explica la configuración de su parque inmobiliario. Según el Censo de Población y Viviendas de 2011 (INE, 2013) había 122.338 viviendas familiares para 102.136 residentes censados, de las que solo $37.6 \%$ eran principales mientras que el $62,45 \%$ eran secundarias o vacías (82.472 unidades residenciales).

El planeamiento del municipio se ha desarrollado de forma caótica sobre la base de multitud de planes parciales que ha imposibilitado cualquier tipo de programa u ordenación del territorio. Se han levantado decenas de urbanizaciones que han colonizado por completo el suelo urbanizable, provocando una alta presión sobre las zonas protegidas y la línea de costa. Este urbanismo desorganizado ha dado origen a una trama viaria enmarañada y confusa, altamente dependiente del vehículo individual, con picos muy elevados de saturación durante la época estival (Aledo y Mazón, 2005). Así, el crecimiento del 
turismo-residencial ha cambiado la morfología urbana del centro urbano de Torrevieja, olvidando el modelo urbano-arquitectónico sismorresistente de la ciudad propuesto en el siglo XIX. No obstante, es cierto que en la zona peri-urbana el modelo urbanístico de urbanizaciones (Casado-Diaz, 1999), al basarse en conjuntos ocupacionales con viviendas de una o dos alturas, con espacios comunitarios amplios y anchos viales, coincide con las propuestas de Larramendi.

Otro punto a discutir, sería la calidad de las construcciones levantadas en los años de los booms inmobiliarios de 1987-1990 y de 1997 a 2007 y su capacidad de soportar movimientos sísmicos potentes. Sin embargo en el casco urbano y en la primera línea de playa, el alto valor del suelo urbano ha provocado un diseño urbano-arquitectónico que maximiza la superficie construida disponible. Los solares, las construcciones de planta baja, las plazas, las calles anchas han sido engullidos por una elevada densidad edificatoria de construcciones en altura. Las calles achicadas por los edificios disminuyen su nivel de seguridad, lo que se complica con el déficit de espacios públicos abiertos y seguros desde el punto de vista de las caídas y desplomes de los edificios y el enmarañado entramado urbano también puede afectar la gestión de la emergencia, dificultando la evacuación o tratamiento de heridos y víctimas (Canales, 1999).

La nueva morfología urbana aumenta no solo la vulnerabilidad arquitectónica sino también la social ante el riesgo sísmico. Los cambios demográficos acaecidos en el municipio provocados por el boom del turismo residencial han ocasionado la aparición de nuevos grupos vulnerables. La llegada de colectivos de migrantes residenciales de edad avanzada e inmigrantes laborales de múltiples nacionalidades y lenguas, junto con elevados picos demográficos como consecuencia de la estacionalidad de las vacaciones (Casado-Diaz, 1999) son factores a tener en cuenta en la producción de vulnerabilidad social.

A partir de los años ochenta, el desarrollo turístico residencial de este municipio ha generado crecimientos exponenciales, duplicando su población cada década. De 25.891 en 1991, se pasó a 58.828 en 2001, hasta alcanzar los 102.136 en 2011 (INE, 2012). Este crecimiento demográfico se debe a la llegada de inmigrantes residenciales y laborales. Según datos del Padrón Municipal en 2011, el 55\% de los residentes censados eran extranjeros, de los que el 23\% proceden del Reino Unido, de Alemania el 7\% y de Rusia el 6\%. Entre los inmigrantes laborales los mayores contingentes proceden de Marruecos que aporta, el $5 \%$, y Colombia, Bulgaria y Rumanía con el $4 \%$ cada uno. Además, el número de personas en el municipio se amplía significativamente en época estival cuando se alcanza, entre residentes y turistas, más de 400.000 personas (Torrevieja, PATECO, 2003). También resulta especialmente relevante el elevado grado de envejecimiento demográfico. El 18\% del total de la población residente tiene entre 50 y 64 años y, un 25\% de los habitantes tiene 65 años o más que corresponde a la alta demanda residencial por parte de jubilados nacionales y de otros países de la Unión Europea (Padrón Municipal, 2011).

Este crecimiento urbanístico no emana de un proceso orgánico o de errores en el planeamiento. Los estudios especializados en el fenómeno turístico residencial español han señalado la evidente conexión entre esta morfología socio-urbanística de rápido crecimiento y nulo planeamiento integral y los intereses de las elites político-empresariales (Auken, 2008; Greenpeace, 2010). El Ayuntamiento de Torrevieja obtenía gran parte de sus ingresos municipales de los diferentes impuestos procedentes de la actividad inmobi- 
liaria por lo que era un impulsor decidido de la política urbanística expansiva. Asimismo, estas elites participaban en operaciones urbanísticas especulativas que les generaban pingües beneficios económicos (Aledo, 2008). En otras palabras el riesgo no se produce solo por la amenaza de un fenómeno natural o por la situación de vulnerabilidad de la población, el riesgo se construye también por la toma de decisiones arriesgadas.

Los intereses económicos también pueden explicar la falta de información y memoria sobre la historia sísmica del municipio. Es significativo que en el Museo de la Mar y la Sal, un espacio, como afirma la web del Ayuntamiento, para «entender los aspectos más relevantes de la historia local» no aparece mención alguna al desastre de 1829. En la página web del Ayuntamiento ${ }^{4}$, tampoco se encuentra información sobre cómo actuar en caso de emergencia sísmica. Asimismo el vigente Plan General de Ordenación Urbana de Torrevieja apenas hace una breve mención al terremoto de 1829 para referenciar el trazado urbano del casco reconstruido como núcleo de interés arquitectónico-urbanístico, pero no lo presenta como un elemento significativo para la ordenación urbanística municipal (PGOU, Torrevieja, 2000: 50).

No obstante, esta situación comienza a cambiar en 2012 con la elaboración del PAM. En las páginas siguientes se describirá y se analizará este plan de riesgo sísmico, bajo la hipótesis que está diseñado y corresponde a la visión dominante de gestión del riesgo y, por lo tanto, no incide con la intensidad necesaria en el análisis y actuación sobre las causas profundas generadoras de vulnerabilidad.

\subsection{Plan de Actuación Municipal (PAM) frente al Riesgo Sísmico de Torrevieja}

El 26 de enero de $2012^{5}$ se forma el grupo de trabajo interdisciplinar ${ }^{6}$ para la producción del PAM. El Plan, coordinador por el Director General de Policía, promueve, entre otras acciones, un ejercicio de simulación de emergencia sísmica en colegios, hospitales y algunas fincas urbanas en marzo de 20137. El PAM se vio impulsado por la nueva legislación en esta materia del Gobierno Valenciano y por sucesos sísmicos acaecidos en 2011. El 13 de enero se aprueba la Estrategia Territorial de la ComunitatValenciana que culmina con la formulación y aprobación del Plan Especial frente al Riesgo Sísmico en la Comunitat Valenciana (Decreto 44/2011). Por otro lado, el 23 de marzo de ese mismo año aconteció en Torrevieja un evento sísmico que alcanzó magnitud 3.1 en la escala Richter pero que tuvo un gran impacto al producirse a las once y media de la mañana en

4 Consulta realizada $<$ www.torrevieja.es $>$, el: 15/05/2013.

5 «Torrevieja asume por primera vez su riesgo sísmico y aborda un plan para hacerle frente. El Ayuntamiento pone en marcha un grupo de trabajo para elaborar un proyecto de actuación municipal en caso de terremoto», Periódico electrónico Información.es, de 27/01/2012.

6 El grupo denominado GT-PAM-RS involucra a la Policía Local, las concejalías de Urbanismo, Vivienda, Sanidad, Obras y Servicios, Alumbrado Público y Ciclo Integral del Agua, Innovación y Nuevas Tecnologías de la Información, además de Protección Civil. Asimismo, prevé la participación de representantes de empresas, instituciones u organismos ajenos al Ayuntamiento, como Cuerpos de Seguridad del Estado, compañías de luz y agua, operadoras de telefonía móvil, colectivos sociales y otras entidades.

7 «Torrevieja se prepara para un terremoto como el de Lorca. El Ayuntamiento y la Generalitat Valenciana realizan el primer simulacro en la provincia frente al riesgo de seísmo», Periódico electrónico Información.es, de 06/03/2013. En este simulacro se incluyó el inglés y el ruso como lenguas operativas en determinadas acciones. 
plena actividad diaria. Consecuencias más dramáticas fueron ocasionadas por el terremoto acaecido en la cercana ciudad de Lorca de 2011 el 11 de mayo, que produjo 9 víctimas mortales (Cabañas et al, 2011). Su alcance destructivo evidenció la necesidad y urgencia del tema, superando el tabú del riesgo sísmico en Torrevieja.

Por lo que respecta a la operatividaddel PAM, los técnicos municipales consultados ${ }^{8}$ señalan que el Plan se basa en cuatro líneas axiales: 1) sensibilización, información y formación; 2) tecnología de comunicación; 3) evaluación técnica; y 4) diagnóstico y movilización de recursos humanos y materiales. En este sentido, el Plan de Actuación Municipal de Torrevieja representa un esfuerzo importante y novedoso. Sin embargo, la preocupación central del PAM es el desarrollo de la estructura organizativa, operativa y de tecnología de información para la gestión de una emergencia por seísmo, sobresaliendo el desarrollo del Programa Base Segura de Emisiones Radioeléctrica (BASEMRAD). Para el coordinador del $\mathrm{PAM}^{9}: \ll$ Nos interesamos por aplicaciones que permitan hacer de teléfonos móviles sencillos y smartphones (Android, principalmente) terminales de teleasistencia con botón de llamada SOS, para que, en caso de grave seísmo, la gente que cuente con estos terminales (miles de usuarios) puedan lanzar SOS de la manera más sencilla y automatizada posible, al tiempo que con la información más completa posible». Hace también parte del proyecto, la construcción de una «Plataforma de Gestión de Ciudad», constituida por un conjunto de aplicaciones que permitirían obtener imágenes del estado de la ciudad en cada momento tras el seísmo.

La preeminencia de este enfoque tecnológico no es exclusiva del PAM de Torrevieja. También puede identificarse en planes de riesgos de escala nacional o regional. Por ejemplo, tanto el Plan Estatal de Protección Civil ante el Riesgo Sísmico (2010) como el Estudio de Vulnerabilidad Sísmica de la Comunitat Valenciana (2011), evalúan la vulnerabilidad sísmica en función de las tipologías de edificación reconocidas, de su antigüedad y de su ubicación geográfica, considerando el nivel de diseño sismorresistente de los edificios y el estado de conocimiento de la época de construcción de la obra, desde la aparición de normas sismorresistentes en España. Se calcula el daño teniendo en cuenta la intensidad del evento sísmico previsto, los años de construcción de las viviendas y el número de habitantes. Por lo tanto no se consideran otras características de la población tales como la edad, el nivel educativo o el tipo de poblamiento, disperso o concentrado, por citar unas pocas. Y al mismo tiempo se da por sentado que la producción de una normativa sismorresistente para los nuevos edificios conlleva su cumplimiento, sin cuestionarse los efectos que pudiera haber tenido en su aplicación el último boom de la construcción en España.

En este sentido, se hace necesario hacer una referencia a la Norma de Construcción Sismoresistente (NSCE-02). Esta norma, que ha experimentado varias revisiones (1974, 1994, 2002) regula la construcción de estructuras sismoresistentes en España. Se aplica a edificios de especial importancia (hospitales, escuelas) y cuando son edificios de importancia normal es obligatoria en aquellas zonas donde se ha calculado una aceleración sísmica superior o igual a 0,08 gr. Es importante señalar que la norma solo regula los elementos estructurales de los edificios.

8 Informaciones obtenidas en entrevista con el, con el Arquitecto Municipal, y con dos miembros de la delegación municipal de Innovación y Nuevas Tecnologías.

9 Director General de Policía, comunicación personal, 01/06/2012. 
Tras el terremoto de Lorca, varios investigadores (Alfaro, et al., 2012; Aretxabala Diez y Sanz Larrea, 2011; Cabañas et al., 2011) han aportado un conjunto de reflexiones críticas sobre esta norma, su implementación y sus deficiencias que son de interés para este caso de estudio.

Se pueden agrupar sus recomendaciones en cuatro áreas.

1. Necesidad de desarrollar mucho más los estudios microzonales para concertar las medidas a los contextos urbanísticos, arquitectónicos y sísmicos locales.

2. Los déficits regulatorios de la propia ley. Debería ampliarse la regulación a los elementos no estructurales (cornisas, antepechos, voladizos) que, como en el caso de Lorca, fueron los que ocasionaron gran parte de los daños y las víctimas

3. El empleo de tipologías constructivas no adaptadas al riesgo sísmico y falta de una cultura sísmica en el sector de la arquitectura y de la construcción.

4. Un mayor control y seguimiento de la aplicación de la normativa ${ }^{10}$.

Señalan Barbat y Pujades que, en las regiones donde se diseña de acuerdo con una normativa sismorresistente adecuada, donde la construcción es sometida a una supervisión estricta y donde el sismo de diseño es representativo de la amenaza sísmica real de la zona, el daño es mínimo en comparación con el que se produce en otras zonas donde no se cumplen estas circunstancias (2004: 233). La oportuna reflexión de estos autores permite introducir un nuevo matiz sobre la visión dominante. Podría afirmarse que es una visión que aisla la regulación de los contextos locales donde se aplica. Pone su fe en la creencia de que con la aprobación de la norma se asegura su aplicación. No reconoce, por tanto, que la regulación es un proceso social del que el dictamen de la ley o norma es solo una fase. Antes, durante y después de la promulgación de la norma hay todo un proceso de negociación, conflicto, resistencia que determina los contenidos y el cumplimiento de los objetivos definidos por la ley.

\section{DISCUSIÓN: LA PERTENENCIA DEL PAM A LA VISIÓN DOMINANTE}

Siguiendo a Phillips y Fordham (2010), la planificación y gestión del riesgo que deviene de la visión dominante se caracteriza por: 1) organizar el modelo de gestión mediante jerarquías y autoridades con un enfoque top-down; 2) abordar la amenaza mediante el uso de la ciencia y la tecnología para dominar/controlar la naturaleza; 3 ) tener como objetivo principal reducir el daño físico; y 4) concentrarse en el proceso físico de la amenaza.

El PAM representa un proyecto pionero en la región y encomiable en su labor de prevención y producción de resiliencia ante el riesgo sísmico. Es de destacar el esfuerzo que

10 Cabe decir a este respecto que a partir de la obligación de contratar por parte de las empresas de un Seguro Decenal y la aparición de los Organismos de Control Técnico (1999) que expiden un certificado de seguridad de la obra necesario para que el promotor pueda contratar el seguro con las compañías aseguradoras, el control de la aplicación de la NSCE-02 que antes recaía exclusivamente en el arquitecto y/o jefe de obra, ha pasado a un control externo que aporta una mayor seguridad. 
supone romper con una tradición de pasividad en la gestión y política del riesgo sísmico por parte de una administración local en un municipio turístico. No obstante un análisis críticamente proactivo del PAM reconocería que su enfoque no resulta equilibrado en cuanto al peso que se le da a la gestión de la vulnerabilidad social. En este sentido es posible apreciar la pertenencia del PAM a la visión dominante. Las causas de este desequilibrio estarían ligadas tanto a la persistencia de la tradición de la visión dominante como a la imposibilidad de cuestionarse las causas profundas que producen el riesgo dado que eso implicaría impugnar el modelo socioeconómico sobre el que se asienta la estructura económica y social del municipio. A continuación se discutirán ambos puntos. Se comenzará por identificar elementos de la visión dominante en la gestión del riesgo que aparecen en el PAM:

1) La persistencia en la planificación del modelo de gestión mediante jerarquías y autoridades con un enfoque top-down. Aunque el PAM incluye la participación de diferentes agentes y actores sociales, no ha habido participación durante su proceso de diseño. Se pide colaboración de diferentes stakeholders para el desarrollo de determinadas acciones (BASEMRAD y Plataforma de Gestión de Ciudad). Sin embargo, la filosofía del plan y las principales acciones a ejecutar no han sido debatidas sino que, diseñadas previamente a la consulta social, han sido impuestas sin una discusión pública sobre la conveniencia de priorizar estas acciones sobre otras que podrían dirigirse a fortalecer la resiliencia de los grupos más vulnerables;

2) El abordaje de la amenaza mediante el uso de la ingeniería, tecnología y la ciencia. Gran parte del esfuerzo del PAM se concentra en la aplicación de TICs para la comunicación y el monitoreo del desastre durante la eclosión del evento sísmico. Estas tecnologías pretenden aportar seguridad y certeza durante un momento de elevado riesgo, incertidumbre y caos mediante la generación y canalización de una enorme cantidad de información dirigida a los claim-makers durante el proceso de toma de decisiones post-desastre. El PAM confía en la capacidad de la tecnología de poner orden en el caos producido por una naturaleza caótica (sea mediante la llegada de información a través de la telefonía móvil o por la visualización de la ciudad durante el terremoto por el sistema de imágenes centralizada). No obstante, surge un escenario de incertidumbre sobre cómo se puede gestionar ese ingente flujo de información que se generaría durante y después del evento sísmico. Por otro lado, en el PAM hay una concentración de esfuerzos en las fases de desastre y post-desastre pero, como se verá seguidamente, son desatendidos factores relevantes generadores de vulnerabilidad y que se situaran en la fase pre-desastre y en las causas de fondo productoras de vulnerabilidad;

3) La reducción del daño físico y la gestión post-desastre como objetivos principales de los programas de gestión del riesgo. Las acciones encaminadas a favorecer la resiliencia y a disminuir la vulnerabilidad social no son objeto prioritario del PAM. Destaca en este sentido que no haya referencias a la vulnerabilidad de la población turística que en temporada vacacional multiplica por cuatro la población censada del municipio; y 
4) El enfoque sobre el proceso físico de la amenaza y en sus consecuencias superficiales. En el PAM no se cuestionan las causas socioeconómicas y urbanísticas profundas que participan en la producción del riesgo; esto es, el hiper-desarrollo del sector turístico residencial que ocasiona elementos de vulnerabilidad tales como el modelo de planeamiento urbanístico o la masificación del municipio durante la temporada alta de verano.

Esta última característica del PAM anuncia un segundo argumento analítico, central en el desarrollo de este trabajo, el de la incuestionabilidad del riesgo. Ni el PAM ni sus redactores -los claim-makers- pueden cuestionar las causas profundas que provocan la producción de vulnerabilidad en Torrevieja en relación con la amenaza sísmica. El riesgo sísmico es desconsiderado en aras a) de proteger el funcionamiento continuo de la maquinaria del crecimiento económico basado en el desarrollo inmobiliario; y b) de promover una gestión cada vez más privatizada del territorio que favorece los procesos de acumulación de las elites políticas y empresariales. Así, el planeamiento fragmentado, erigido sobre decenas de planes parciales, se adapta a las necesidades del promotor pero dificulta un enfoque integral del desarrollo urbanístico del municipio. El cuestionamiento de este modelo urbano debería ser requisito imprescindible para implementar programas de gestión de riesgos ya que se lograría identificar una de las causas profundas de producción de vulnerabilidad: la errónea planificación urbanística ante el riesgo sísmico. Por su parte, el papel de la ciencia es crítico en este proceso porque se presenta por parte de los claim-makers como el agente capaz de generar, en un siempre aplazable futuro, certezas y de proporcionar control sobre la naturaleza. En ese sentido, la ciencia y la tecnología funcionan como ansiolíticos sociales que ofrecen seguridad en escenarios de alta incertidumbre y riesgo.

En la era del postecologismo, las actuaciones socioambientales -léase el PAM- ya no se encaminan a producir sustentabilidad sino a manejar la insostenibilidad a fin de mantener un orden socio-ecológico precario pero que faculte a las elites a seguir imponiendo sus procesos de acumulación de riqueza -léase el modelo turístico residencial español-. Por lo tanto, como apuntan Blühdorn y Welsh (2007), resulta imposible sistémicamente que los análisis de riesgo analicen las causas profundas de su origen. Si eso se llevara a cabo se cuestionarían los principios imperativos del actual modelo socioeconómico global hiperconsumista. El cuestionamiento del riesgo supondría impugnar el orden social, contradecir a las elites locales y nacionales e impugnar el propio papel de los gestores tecno-científicos del riesgo.

Por un lado, la población envejecida, población extrajera que no dominan o hablan el castellano, veraneantes y residentes extranjeros que no conocen la historia sísmica del municipio son grupos vulnerables que no reciben una especial atención en el PAM, ni se han diseñado programas de prevención y actuación ante el riesgo sísmico para estos colectivos. Por otro lado, el planeamiento urbanístico y el entorno arquitectónico del casco urbano de Torrevieja contradicen el pasado sísmico del municipio y genera unas condiciones urbanísticas de alto riesgo. La razón de estas deficiencias está relacionada con el desarrollo turístico residencial, sector sobre el que se construye la pirámide social y económica de este municipio. Incidir sobre los grupos vulnerables y sobre las causas profundas del 
riesgo sería cuestionar el modelo de turismo residencial implementado en Torrevieja. Sería cuestionar su propuesta de crecimiento ilimitado. Y sería impugnar la lógica mercantilista que ha condicionado las decisiones sobre el planeamiento del territorio ${ }^{11}$.

En el caso del PAM, las decisiones que se acuerdan en la gestión del riesgo, se dirigen sobre el control o manejo tecno-científico de la amenaza y a intentar contraer o minimizar las condiciones más superficiales y locales de la vulnerabilidad (condiciones inseguras según la terminología de Wisner et al., 2004: 30). En definitiva, los factores de producción de vulnerabilidad y riesgo relacionados con desarrollos urbanísticos no sostenibles son esquivados pues pondría en cuestión el modelo de acumulación por parte de las elites locales y regionales.

\section{CONCLUSIÓN: LA CUESTIONABILIDAD DEL RIESGO}

El estudio de caso seleccionado ha posibilitado ilustrar el predominio de la visión dominante en la gestión de los riesgos ambientales y ejemplificar el concepto de incuestionabilidad del riesgo. De esta manera, el análisis de la gestión del riesgo sísmico en un municipio turístico-residencial sirve para reflejar la contradicción de la sociedad del riesgo: cómo una sociedad se enfrenta, por un lado, a la probabilidad de un desastre socionatural y, por otro, a la imposibilidad de cuestionar las bases, el modo de producción, que genera vulnerabilidad y contextos de riesgo global.

Algunos expertos (Hewitt, 1998; Mileti, 1999; Wisner, 2000) han dudado de que dentro del actual sistema socioeconómico capitalista, neoliberal, globalizado e hiperconsumista pueda llevarse a cabo la integración adecuada del enfoque de la vulnerabilidad en la gestión de los riesgos ambientales debido a que los factores activos en las causas profundas son incuestionados. Por el contrario, no ha sido la intención de este artículo la de argumentar la incuestionabilidad del riesgo para justificar una posición inhibidora o paralizante.

Para el caso que ha ocupado este artículo, las propuestas de actuación sobre las causas profundas irían orientadas a transformar el actual modelo de gestión urbanística en municipios turístico-residenciales hacia modelos más viables y más democráticos. Sobre este tema ya existe una suficiente bibliografía que incide en temas de gobernanza (Alcaraz, 2007; Fernández, 2007; Fundación Alternativa, 2009; GRECO, 2011), diversificación económica (Exceltur, 2005; García-Andreu, 2008) y ordenación del territorio y protección ambiental (Vera Rebollo, 2003; Greenpeace, 2005, 2010). Asimismo, dentro de la literatura sobre la gestión del riesgo se propone la combinación de la aproximación de las causas profundas con la importancia de un enfoque intermedio que se denomina Compensatory Disaster Risk Management (UNISDR, 2011). Esta estrategia se centra en actuaciones de prevención y mitigación ante riesgos inmediatos y manejando escalas meso y locales. Las líneas generales de este enfoque estarían orientadas a: 1) identificación de grupos vulnerables y diseño de medidas de prevención y mitigación específicas para estos grupos; 2) la información y formación sobre el comportamiento en caso de

11 Situación muy similar refleja Calvo García Tornel y Granell Pérez (2009) sobre el riesgo de inundaciones en el Sureste Español. 
terremotos tanto a la población en general, como a los grupos más vulnerables y a los turistas; 3) aplicación estricta de las medidas sismorresistentes en la construcción y recuperación del modelo de Larramendi en los futuros desarrollo inmobiliarios.

El propósito final de este artículo es revelar la incuestionabilidad del riesgo como paso obligado para construir un paradigma de la vulnerabilidad que integre el abordaje de las causas profundas; en otras palabras, su cuestionamiento. Se puede encontrar soportes para ese proceso reflexivo, enriquecido con fórmulas participativas (Funtowicz y Ravetz, 2000), en el propio corpus científico institucionalizado de la economía urbana y ambiental (Marínez Alier, 2009; Tojo y Naredo, 2010), de la geografía (Calvo García-Tornell, 1997), de la sociología (Beck, 1992) o de las humanidades (Ehrenfeld, 1978). El enfoque de la vulnerabilidad conlleva una nueva gestión del riesgo sísmico, concebida como un recurso y no como un coste. A causa de la especulación inmobiliaria y con la connivencia de las autoridades públicas que han basado sus presupuestos en los rendimientos de la actividad inmobiliaria, en Torrevieja se ha olvidado la amenaza sísmica. La mención al riesgo sísmico era entendida como un posible freno al desarrollo económico del municipio pues podría amedrentar a futuros residentes. Sin duda, un factor clave para este nuevo enfoque del riesgo sísmico en municipios turísticos pasa obligadamente por su inclusión en la gestión actual y futura de la ciudad, por el abordaje preventivo a través de la comunicación social y por la implementación de planes y programas de preparación ante este riesgo para que se construya seguridad y confianza a la ciudadanía.

\section{BIBLIOGRAFÍA}

ABRAMOVITZ, J.N. (2001): Unnatural Disasters. Worldwatch Paper 158. Washington, Worldwatch Institute.

ALCARAZ, M. (2007): El Estado de Derecho frente a la Corrupción Urbanística. Madrid, La Ley.

ALEDO, A. (2008): «De la tierra al suelo: la transformación del paisaje y el Nuevo Turismo Residencial», Arbor: Ciencia, Pensamiento y Cultura, vol 184, n 729, pp. 99-113.

ALEDO, A y MAZÓN, T. (2005): Turismo residencial y cambio social: nuevas perspectivas teóricas y empíricas. Alicante, Universidad de Alicante - CAM.

ALEDO, A.; MAZÓN, T. y MANTECÓN, A. (2007): «La insostenibilidad del turismo residencial», en Antropología y Turismo: claves culturales y disciplinares. México, Plaza y Valdés, pp. 185-208.

ALEXANDER, D. (2000): Confronting Catastrophe. Hertfordshire Terra.

ALFARO, P. et al. (2011): «Lecciones aprendidas del terremoto de Lorca de 2011»; en Enseñanza de las Ciencias de la Tierra, vol. 19, n 3, pp. 245-260.

ARETXABALA DIEZ, A.; SANZ LARREA, C. (2011): Why was it so damaging?, en Multimedia Technology (ICMT), $20112^{\text {ND }}$. International Conference On. Hangzhou, pp. 6670-6679.

AUKEN, M. (2008): Report on the Impact of Extensive Urbanisation in Spain on Individual Rights of European Citizens, on the Environment and on the Application of EU Law, based upon Petitions received European Parliament. 
AYALA-CARCEDO, F.J. y OLCINA J. (2002): «Riesgos naturales. Conceptos fundamentales y clasificación», en Riesgos naturales. Barcelona, Ariel, pp. 41-74.

BANKOFF, G.; FRERKS, G. y HILHORST D. (2004): Mapping vulnerability: Disasters, Development, and People. Sterling, Earthscan.

BARA, C. (2010): Factsheet: social vulnerability to disasters. Center for Security Studies (CSS), ETH Zurich.

BARBAT, A y PUJADES, L. (2004): «Evaluación de la Vulnerabilidad y del riesgo sísmico en zonas urbanas. Aplicación a Barcelona», en Sísmica 2004: $6^{\circ}$ Congresso Nacional de Sismologia e Engenharia Sísmica. pp. 229-252.

BAUMAN, Z. (1996): «Modernidad y ambivalencia», en Las consecuencias perversas de la modernidad. Barcelona, Anthropos, pp. 73-119.

BECK, U. (1992): Risk Society: Towards a New Modernity. Londres, SAGE Publications.

BLANCHARD, W. (2000): Higher Education Project Presentation at FEMA Emergency Management Institute. Emmitsburg, MD.

BLÜHDORN, I. (2000): Post-ecologist politics: social theory and the abdication of the ecologist paradigm. Nueva York, Routledge.

BLÜHDORN, I. (2009): «Narratives of Self-Delusion: Towards a Critical Theory of the Politics of Unsustainability», Conference paper presented at the 2009 Annual Meeting of the American Political Science Association. Toronto, Canada.

BLÜHDORN, I. y WELSH, I. (2007): «Eco-politics beyond the Paradigm of Sustainability», Environmental Politics, vol. 16, n 2, pp. 185-205.

BRYANT, R. L. y BAILEY, S. (1997): Third World Political Ecology. Routledge.

BURTON, I.; KATES, R.W. y WHITE, G.F. (1993): The Environment as Hazard. Nueva York, The Guilford Press.

CABAÑAS, L.; CARREÑO, E.; IZQUIERDO, A.; MARTÍNEZ, J.M.; CAPOTE DEL VILLAR, R.; MARTÍNEZ, J.; BENITO, B.; GASPAR, J.; RIVAS, A.; GARCÍA, J.; PÉREZ, R.; RODRÍGUEZ, M.A. y MURPHY, P. (2011): Informe del sismo de Lorca del 11 de mayo de 2011. Madrid, Instituto Geográfico Nacional.

CALVO GARCÍA-TORNEL, F. (19977): «Algunas cuestiones sobre Geografía de los riesgos», Scripta Nova. Revista Electrónica de Geografía y Ciencias Sociales, vol. 1, nº 1-13.

CALVO GARCÍA-TORNEL, F. y CANALES MARTÍNEZ , G. (2009): «Una planificación urbanística antisísmica en el siglo XIX y su evolución posterior», Murgetana $\mathrm{n}^{\circ}$ 121, pp. 191-208.

CALVO GARCÍA-TORNEL, F y GRANELL PÉREZ, M.C. (2009): «Valoración social del riesgo por inundación en el litoral meridional de la Región de Murcia», Scripta Nova. Revista Electrónica de Geografía y Ciencias Sociales. Barcelona: Universidad de Barcelona, vol. XIII, núm. 295. Disponible en <http://www.ub.es/geocrit/sn/sn-295.htm>.

CANALES MARTÍNEZ, G. (1999): La catástrofe sísmica de 1829 y sus repercusiones. Murcia, Pictografía.

CANNON, T. (1994): «Vulnerability Analysis and the Explanation of 'Natural' Disasters», en Disasters, Development and Environment. Londres, John Wiley, pp. 13-30.

CASADO-DIAZ, M.A. (1999): «Socio-demographic Impacts of Residential Tourism: a Case Study of Torrevieja, Spain», International Journal of Tourism Research, vol. 1, $n^{\circ} 4$, pp. 223-237. 
CUTTER, S.L. (1996): «Vulnerability to environmental hazards», en Progress in Human Geography, vol. 20, n 4, pp. 529-539.

DRYZEK, J.S. (2005): The politics of the earth: environmental discourses. Oxford University Press.

EHRENFELD, D. (1978): The arrogance of the Humanism. Nueva York, Oxford University Press.

ENARSON, E.; CHILDERS, C.; HEARN, B.; THOMAS, D. y WISNER, B. (2003): A Social Vulnerability Approach to Disasters. FEMA Emergency Management Institute. Emmitsburg, Maryland.

EXCELTUR (Alianza para la excelencia turística) (2005), «Impacto de los modelos de desarrollo turístico en el litoral mediterráneo y las islas», 22 de agosto de 2013. Madrid. Disponible en http://exceltur.org/excel01/contenido/portal/files/auxfolder3/ Sumario_residencial_completo.pdf

FERNÁNDEZ, G. (2007): «Las peculiaridades del modelo urbanístico español: la especulación legalizada del suelo», Documento presentado en el curso Algunas Claves de la Corrupción Urbanística en España. Fundación César Manrique. Disponible en http:// www.fcmanrique.org/nota.php?idNoticia $=92$

FUNDACIÓN ALTERNATIVA. (2009): Informe sobre la Democracia en España. Madrid, Fundación Alternativa.

FUNTOWICZ, S. y RAVETZ, J.R. (2000): La ciencia posnormal: ciencia con la gente. Barcelona, Icaria Editorial.

GARCÍA-ANDREU, H. (2008): Sociedad, Turismo y Medio Ambiente: una propuesta desde la Sociología para el diagnóstico y reorientación de los municipios turísticoresidenciales del litoral español (Society, Tourism and Environment: A Proposal for the diagnosis and reorientation of residential tourism municipalities in the spanish coast). $\mathrm{PhD}$ dissertation in sociology, University of Alicante, Spain.

GEORGESCU-ROEGEN, N. (1971): The entropy law and the economic process. Cambridge, Harvard University Press.

GIDDENS, A. (1990): The consequences of modernity. Cambridge, Polity Press.

GILBERT, C. (1995): «Studying Disaster: A Review of the Main Conceptual Tools», International Journal of Mass Emergencies and Disasters, vol. 13, $\mathrm{n}^{\circ}$ 3, pp. 231-240.

GINER, J. J., MATEO, P.A. y ESTÉVEZ, A. (1992): Peligrosidad sísmica en el término municipal de Torrevieja: aproximación a la evaluación del riesgo sísmico. Memoria informativa. Universidad de Alicante, Ayuntamiento de Torrevieja.

GINER, J.J. (1996): Sismicidad y Peligrosidad Sísmica en la Comunidad Autónoma Valenciana. Tesis Doctoral, Universidad de Granada. Disponible en

http://web.ua.es/es/urs/peligrosidad/mapas-de-peligrosidad-sismica-en-la-c-v-modelosprobabilisticos.html.

GROUPE D'ETATS CONTRE LA CORRUPTION (GRECO). (2011): Compliance Report on Spain: Incriminations (ETS 173 and 191, GPC 2): Transparency of Party Funding. Greco RC-III (2011) 5E. Strasbourg, GRECO Secretariat Council of Europe.

GREENPEACE. (2005): «Destrucción a toda Costa 2005», 19 de marzo de 2011. Disponible en http://www.greenpeace.org/espana/Global/espana/report/other/destrucci-na-toda-costa-2005-12.pdf 
GREENPEACE. (2010): «Destrucción a toda Costa 2010», 4 de mayo de 2011. Disponible en http://www.greenpeace.org/espana/Global/espana/report/other/100709-04.pdf

HEWITT, K. (1983): «The Idea of Calamity in a Technocratic Age», en Interpretations of Calamity. Boston, Allen and Unwin, pp. 3-32.

HEWITT, K. (1998): «Excluded Perspectives in the Social Construction of Disaster», en What is a Disaster? Perspectives on the Question. Nueva York, Routledge, pp. 75-91.

JUNEJA, S. (2009): «Disasters and Poverty: The Risk Nexus. A Review of Literature», Background Paper for the 2009 ISDR Global Assessment Report on Disaster Risk Reduction.

KLINENBERG, E. (2002): Heat Wave: A Social Autopsy of Disaster in Chicago. Chicago, University of Chicago Press.

LARAÑA, E. (1999): «Modernización, reflexividad y riesgo», en Sociología y Medio Ambiente: estado de la cuestión. Madrid, Fundación Fernando de los Ríos-Universidad Pública de Navarra.

LARRAMENDI, J.A. (1829a): Memoria y relación circunstanciada de los estragos que la terrible catástrofe de los terremotos de 21 de marzo y siguientes, principalmente el del Sábado Santo 18 de abril hasta el presente día, han causado en Torrevieja y demás pueblos de la gobernación de Orihuela y sus inmediaciones, en la ciudad de Murcia y algunos pueblos de la provincia de este nombre. Madrid, de orden Superior, Imprenta Real, p. 5.

LARRAMENDI, J.A. (1829b): Exposición de José Agustín Larramendi al Excelentísimo Señor Don Manuel González Salmón. Orihuela, Sección Estado, legajo no 3.173 , Archivo Histórico Nacional, Madrid, mayo.

LÁZARO, R. (1829): Relación que manifiesta el número de casas y edificios de estas poblaciones de La Mata y Torrevieja que se hallan arruinadas al todo, en parte y quebrantadas, el número de muertos, con expresión de hombres, mujeres y niños; y el de heridos y contusos por causa de los terremotos del día 21 de marzo último. Torrevieja, 27 de junio. Correspondencia de Policía y Terremotos, signatura D. 172 (1829). Fondos Municipales. Archivo Histórico de Orihuela.

MARTÍNEZ-ALIER, J. (2009): El ecologismo de los pobres: conflictos ambientales y lenguajes de valoración. Barcelona, Icaria.

McENTIRE, D. A. (2003): «Causation of catastrophe: lesson from Hurricane George», Journal of Emergency Management, vol. 1, $\mathrm{n}^{\mathrm{o}}$ 2, pp. 22-29.

MILETI, D. (1999): Disasters by Design: A Reassessment of Natural Hazards in the United States. Washington, DC, Joseph Henry Press.

MILLENNIUM ECOSYSTEM ASSESSMENT. (2005): Ecosystems and Human Wellbeing: General Synthesis. Washington, Island Press.

PLAN DE ACCIÓN TERRITORIAL APLICADO A LA DISTRIBUCIÓN COMERCIAL DE TORREVIEJA (PATECO). (2003). Torrevieja, Ayuntamiento de Torrevieja.

PLAN GENERAL DE ORDENACION URBANA DE TORREVIEJA (PGOU). (2000). Torrevieja, Ayuntamiento de Torrevieja.

PELIGROSIDAD SÍSMICA EN LA COMUNITAT VALENCIANA (PELSISCAV). (2010). Valencia, Generalitat Valenciana. 
PELLING, M. (2003) PELLING, Mark. The vulnerability of cities: natural disasters and social resilience. Londres, Earthscan.

PHILLIPS, B. y FORDHAM, M. (2010): «Introduction», en Social Vulnerability to Disasters. Nueva York, CRC Press, pp. 1-23.

QUARANTELLI, E.L. (1985): «What Is Disaster? The Need for Clarification in Definition and Conceptualization in Research», en Disasters and Mental Health: Selected Contemporary Perspectives. U.S. Department of Health and Human Services, National Institute of Mental Health, pp. 41-73.

QUARANTELLI, E. L. (1998): What is a Disaster? Londres, Routledge.

RENN, O. (2008): Risk Governance: Coping with Uncertainty in a Complex World. Londres, Earthscan.

ROBBINS, P. (2004): Political ecology: a critical introduction. Blackwell Publishing.

SCANDLYN, J.; SIMON, C.N.; THOMAS, D. y BRETT, J. (2010): «Theoretical Framing of Worldviews, Values, and Structural Dimensions of Disasters», en Social Vulnerability to Disasters. Nueva York, CRC Press, pp. 27-49.

SCHNAIBERG, A. (1980): The Environment: From Surplus to Scarcity. Nueva York, Oxford University Press.

SHELDRAKE, R. (1994): El renacimiento de la naturaleza. El resurgimiento de la ciencia y de Dios. Barcelona, Editorial Paidós.

STALlingS, R. A. (1995): Promoting Risk: Constructing the Earthquake Threat. Hawthorne, NY, Aldine de Gruyter.

STEINBERG, T. (2000): «Disaster as Archetype», en Acts of God: The Unnatural History of Natural Disaster in America. Nueva York, Oxford University Press, pp. 25-46.

TIERNEY, K.J. (2007): «From the margins to the mainstream? Disaster research at the crossroads», Annual Review of Sociology, vol. 33, pp. 503-525.

TOBIN, G. y MONTZ, B. (1997): Natural Hazards: Explanation and Integration. Nueva York, Guilford.

TOJO, J. y NAREDO, J.M. (2010): Libro blanco de la sostenibilidad en el planeamiento urbanístico español. Madrid, Ministerio de Vivienda.

UNITED NATIONS DEVELOPMENT PROGRAMME (UNDP). (2004): Reducing Disaster Risk: A Challenge for Development. Nueva York, UNDP.

UNITED NATIONS OFFICE FOR DISASTER RISK REDUCTION (UNISDR) Programa de Reducción de Desastres de Naciones Unidas. (2009) Terminología sobre reducción del riesgo de desastres, Ginebra, Naciones Unidas.

UNITED NATIONS OFFICE FOR DISASTER RISK REDUCTION (UNISDR). (2011): Revealing risk, redefining development: global assessment report on Disaster Risk Reduction. United Nations International Strategy for Disaster Reduction. Geneva.

VERA REBOLLO, J.F. (2003): «Riesgos naturales en la actividad turística», Areas: Revista Internacional De Ciencias Sociales, $\mathrm{n}^{\circ} 23$, pp. 159-175.

WISNER, B. (2000): «Capitalism and the Shifting Spatial and Social Distribution of Hazard and Vulnerability», Australian Journal of Emergency Management, vol. 16, $\mathrm{n}^{\mathrm{o}} 2$, pp. 44-50.

WISNER, B., BLAIKIE, P., CANNON, T. y DAVIS, I. (2004): At Risk: Natural Hazards, People's Vulnerability and Disasters. Londres, Routledge. 
